\title{
Influence du poids à 10 jours et de l'âge à la castration des porcelets mâles sur la croissance et la qualité des carcasses à l'abattage
}

\author{
B Desmoulin† ${ }^{\dagger}$ A Aumaître*, J Peiniau \\ avec la collaboration de J Lebost, G Conseil, JC Hulin, \\ $P$ Ecolan, L Jaffrennou, A Barbeau \\ INRA, centre de recherches de Rennes, Station de recherches porcines, \\ Saint-Gilles, 35590 L'Hermitage, France
}

(Reçu le 18 avril 1990, accepté le 27 septembre 1990)

\begin{abstract}
Résumé - On a comparé chez le porcelet mâle à la fois l'effet du poids à $10 \mathrm{j}(2,7$ contre $3,6 \mathrm{~kg})$ et celui de l'âge à la castration (10 et $21 \mathrm{j}$ ) sur la vitesse de croissance, l'efficacité alimentaire de la ration et la proportion de tissus maigres et gras dans la carcasse à $100 \mathrm{~kg}$, par rapport aux animaux entiers. Cent vingt animaux, issus de 40 portées, ont été utilisés, répartis selon le schéma des blocs incomplets équilibrés de type 1 répété 2 fois pour les 6 traitements. La vitesse de croissance jusqu'au sevrage est affectée par le poids initial à la naissance ou à $10 \mathrm{j}$ : l'âge à $25 \mathrm{~kg}$ diffère significativement de $4,5 \mathrm{j}$, celui à $100 \mathrm{~kg}$ de $6 \mathrm{j}(P<0,01)$ entre les animaux lourds et légers. De plus, les porcelets les plus légers à $10 \mathrm{j}$ contiennent une proportion de muscle significativement plus faible que celle des animaux lourds. La castration entraîne, quel que soit l'âge auquel elle est pratiquée, une réduction des performances de croissance, une diminution de la proportion de viande maigre de 51,9 à $48,6 \%$ en moyenne, et une augmentation de la porportion de gras de la carcasse à l'abattage. La quantité d'aliment consommé à $100 \mathrm{~kg}$ diffère de $35 \mathrm{~kg}$ entre mâles entiers et mâles castrés; l'indice de consommation est réduit de $15 \%(2,77$ contre 3,19$)$. La castration à $10 \mathrm{j}$ est suivie d'une réduction significative de la croissance après sevrage des animaux. Elle réduit surtout (bien que non significativement) de 1 point de 49,1 à $48,1 \%$ la proportion de muscle et augmente de 28,7 à $29,6 \%$ la proportion de tissus gras par rapport à la castration à $21 \mathrm{j}$. Les risques d'une castration trop précoce des porcelets mâles sur leurs performances sont discutés.
\end{abstract}

porc mâle / castration / âge / croissance / carcasse

Summary - Influence of weight at $10 \mathrm{~d}$ and age at castration in male piglets on growth rate and carcass quallty. The effects of weight at the age of $10 \mathrm{~d}(2.7 \mathrm{vs} 3.6 \mathrm{~kg})$ and age at castration (10 and 21 d) on growth rate, feed efficiency and the amount and percentage of fat and lean meat in the carcass were tested in comparison with entire males Large White pigs. One hundred and twenty animals issued from 40 litters were affected to 6 experimental treatments according to the incomplete bloc design involving 3 animals in a litter, and repeated twice. Growth rate until weaning depended on the initial weight at birth or at $10 \mathrm{~d}$. The age at $25 \mathrm{~kg}$ and $100 \mathrm{~kg}$ liveweight, respectively differed significantly ( 4.5 and $6 d$ respectively; $\mathrm{P}<0.01$ ) between heavy and light animals. Moreover,

* Correspondance et tirés à part 
the lighest animals at $10 \mathrm{~d}$ contained a significantly smaller proportion of muscle than heavy animals. Whatever the age it was carried out, castration induced a decrease in growth performance, a reduction in the average percentage of lean meat (48.6\% vs $51.9 \%$ ) and an increase in the proportion of carcass fat at slaughter. The feed intake at $100 \mathrm{~kg}$ varied significantly between entire and castrated males, and food conversion ratio was reduced by $15 \%$ in entire males (2.77 vs 3.19 ). Castration at $10 \mathrm{~d}$ reduced significantly post weaning growth rate. The possible disadvantages of early castration on male light piglets performance are discussed, especially the tendency toward the reduction of the proportion of lean meat in the carcass.

male pig / castration / age / growth / carcass

\section{INTRODUCTION}

En Europe continentale, les porcelets mâles sont généralement castrés peu après la naissance. Des données déjà anciennes montrent que cette pratique est sans conséquence sur la croissance pendant l'allaitement et jusqu'à 2 mois d'âge (Salmon-Legagneur et Février, 1959; Charrette, 1961). Mais elle se traduit cependant par une réduction de la rentabilité de l'élevage en raison d'une élévation de l'indice de consommation et d'une diminution significative du pourcentage de muscles de la carcasse (Prescott et Lamming, 1967; Pay et Davies, 1973; Desmoulin et Bonneau, 1979; Walstra, 1980).

La supériorité de la vitesse de croissance des porcs mâles entiers sur les animaux castrés observée par Balun (1973) ou Bublik et Gerasimov (1977) ne semble pas être confimée dans toutes les conditions d'alimentation (Tesanovic et Jovicic, 1975; Desmoulin et Bonneau, 1979; Walstra, 1980; Knudson et al, 1985). En revanche, la castration entraîne toujours, par rapport à l'animal entier, une augmentation des dépôts gras, mesurée soit par l'épaisseur de lard, soit par la proportion de morceaux maigres et de morceaux gras (Charette, 1961; Pay et Davies, 1973; Desmoulin et Bonneau, 1979). Par ailleurs, on a observé que plus la castration est tardive, plus l'épaisseur de lard à $100 \mathrm{~kg}$ est réduite (Charette, 1961; Jost, 1971; Domanski, 1973). Mais la castration à $80 \mathrm{~kg}$ considérée comme stade optimal par certains auteurs (Warwick et Van Lone, 1926; Grosse, 1963) est une opéra- tion traumatisante impossible à généraliser en élevage.

L'effet d'une castration précoce n'a jamais été étudié en relation avec le poids de l'animal au moment de l'opération : on a seulement observé qu'une castration dès la naissance peut réduire la vitesse de croissance sur l'ensemble de la période (Bublik et Gerasimov, 1977; Gonzales et al, 1984). D'autres observations basées sur le rôle des stéroïdes sexuels sur le contrôle de l'adiposité chez le porc permettent de recommander une castration très tardive, vers 20 semaines d'âge, pour bénéficier d'une croissance maximale et conduire à la production d'une carcasse aussi maigre que celle des animaux entiers (Grosse, 1963). Par ailleurs, une modification de la courbe de croissance pendant le jeune âge liée soit au poids à la naissance, soit au niveau alimentaire, entraîne des répercussions à long terme sur les performances pondérales et la proportion de tissus maigres de la carcasse.

La présente expérience a été entreprise en vue de vérifier si la castration des porcelets à 10 ou à $21 \mathrm{j}$, différant par le poids à la naissance et à $10 \mathrm{j}$, modifie les performances des animaux.

\section{MATÉRIEL ET MÉTHODES}

\section{Animaux}

Cent vingt porcelets de race Large White issus de 40 portées, comportant chacune au moins 3 animaux mâles ont été sélectionnés à l'âge de $10 \mathrm{j}$ pour satisfaire un dispositif expérimental 
permettant de comparer 6 traitements. Les animaux sont répartis alternativement à raison de 3 ¿frères de portée «légers" $(1,96-3,0 \mathrm{~kg})$, puis $3 \AA$ frères de portée "courds" $(3,2-4,73 \mathrm{~kg})$ issus respectivement de 2 portées contemporaines à l'un des 6 traitements. La constitution d'un bloc est effectuée selon le schéma des blocs incomplets équilibrés de type I (Cochran et Cox, 1957). Dix blocs sont ainsi constitués comprenant 1 mâle entier lourd, 1 léger à 10 j; 1 mâle léger et 1 mâle lourd castrés à 10 j; 1 mâle léger et 1 mâle lourd castrés à 21 j et ce dispositif est répété 2 fois afin d'augmenter la puissance du test. Les porcelets sont élevés en groupe par la mère jusqu'au sevrage vers $23 \pm 1 \mathrm{j}$. Ils sont ensuite logés individuellement jusqu'à l'abattage. Ils reçoivent un aliment de sevrage pendant 4 semaines, puis un aliment $2^{\mathrm{e}}$ âge entre $49 \mathrm{j}$ et le poids de $27 \mathrm{~kg}$ environ. Ils sont transférés en loge individuelle de croissance-engraissement aux environ de $27 \mathrm{~kg}$ et jusqu'à l'abattage, aux environs de $100 \mathrm{~kg}$ de poids vif, ils reçoivent le même aliment distribué à volonté pendant toute la période (tableau I).

\section{Mesures}

Les animaux sont pesés à la naissance $(0-8 \mathrm{~h}$ après), à $10 \mathrm{j}$, au sevrage, puis chaque semaine ensuite jusqu'au poids vif final de $100 \mathrm{~kg}$. Les performances moyennes sont exprimées pour les périodes suivantes : naissance au sevrage; sevrage à $25 \mathrm{~kg} ; 25-60 \mathrm{~kg}$ (croissance); $60-100$ $\mathrm{kg}$ (finition). L'âge à $25 \mathrm{~kg}$ est calculé par régression en tenant compte du poids observé lors des 2 pesées encadrant cette performance. Les quantités individuelles d'aliment consommé

Tableau I. Composition centésimale et chimique des aíinents en fonction du stade des animaux.

\begin{tabular}{lccc}
\hline $\begin{array}{l}\text { Aliment et } \\
\text { période }\end{array}$ & $\begin{array}{c}\text { Sevrage } \\
(21-49 j)\end{array}$ & $\begin{array}{c}2^{\circ} \text { âge } \\
(49 j-27 \mathrm{~kg})\end{array}$ & $\begin{array}{c}\text { Croissance-finition } \\
(27-100 \mathrm{~kg})\end{array}$ \\
\hline Composants: & - & 21 & 23 \\
Blé & - & 28,5 & 18 \\
Maïs & 53,7 & 10 & 24 \\
Orge & - & - & 7 \\
Son de blé & - & - & 3 \\
Mélasse & 4 & - & - \\
Suif 1er jus & 19 & - & 20 \\
Tourteauu de soja & 3 & - & - \\
CPSP (90) & 15 & 1,5 & - \\
Poudre de lait écrémé & - & 1,8 & 1,7 \\
Poudre de lactosérum & 1,6 & 0,45 & 1,7 \\
Carbonate de calcium & 2,4 & 1,35 & 0,45 \\
Phosphate bicalcique & 0,3 & & 1,15 \\
Chlorure de sodium & 1 & 87,65 & \\
Oligoéléments, vitamines & & 19,67 & 88,58 \\
& & 6,77 & 17,60 \\
Analyse chimique : & 88,51 & 3,36 & 6,49 \\
Matière sèche & 20,72 & & 2,95 \\
Protéines (N x 6,25) & 7,90 & & \\
Cendres & 2,80 & & \\
Cellulose brute (Weende) & & & \\
\hline
\end{tabular}

${ }^{1}$ Concentré de protéines solubles de poisson, $90 \%$ de protéines. 
sont mesurées tous les jours et exprimées aux périodes définies précédemment. A l'abattage, on mesure le poids net de la carcasse sans tête, l'épaisseur minimale de lard dorsal au rein sur la fente, l'épaisseur du gras latéral à $8 \mathrm{~cm}$ entre les $3^{\theta}$ et $4^{\theta}$ vertèbres lombaires, l'épaisseur du gras latéral à $6 \mathrm{~cm}$ à 3 vertèbres en arrière de la dernière côte et l'épaisseur de muscle au même niveau. Les mesures linéaires d'épaisseur de lard et de muscle sont effectuées à l'aide d'une sonde Fat-O-Meater (FOM, SFK Copenhague, Danemark). Le lendemain de l'abattage, les carcasses sont découpées selon une procédure normalisée (Desmoulin et al, 1984). Les pourcentages de muscle et de gras de la carcasse sont calculés soit à partir des mesures linéaires, soit à partir des pièces de découpe, à l'aide des équations décrites par Desmoulin et al (1988).

\section{Analyses statistiques}

Les résultats moyens sont comparés entre traitements par une analyse de variance appliquée aux blocs incomplets équilibrés de type I grâce a la procédure SAS. Les principaux facteurs analysés sont le poids à $10 \mathrm{j}(P)$, la castration (C), et l'interaction poids $x$ castration $(P \times C)$. L'effet de l'âge à la castration $(A)$ est testé par la méthode des contrastes entre 10 et $21 \mathrm{j}$ (traitements 2 et 5 contre traitements 3 et 6 ).

\section{RÉSULTATS}

\section{Performances zootechniques}

Les poids moyens observés à âge fixe (tableau II) montrent des différences significatives par définition à $10 \mathrm{j}$, et des différences variant dans le même sens à la naissance et au sevrage. La croissance journalière entre $10 \mathrm{j}$ et le sevrage (fig ta et tableau II) diffère de $23 \%$ entre les animaux lourds et légers. Enfin, au cours des 2 semaines qui suivent le sevrage, la vitesse de croissance des porcelets (légers et lourds), castrés à 10 j est diminuée, mais non significativement (fig 1b) par rapport à celle des animaux entiers ou castrés à $21 \mathrm{j}$.

Pendant la période globale qui sépare le sevrage du poids de $27 \mathrm{~kg}$, le gain de poids moyen ne diffère pas significativement entre traitements, les animaux présentant des performances très homogènes. La quantité d'aliment consommé est semblable dans tous les traitements; l'indice de consommation est toutefois augmenté chez les animaux castrés lourds castrés à $21 \mathrm{j}$.

La vitesse de croissance des animaux mâles entiers est semblable à celle des

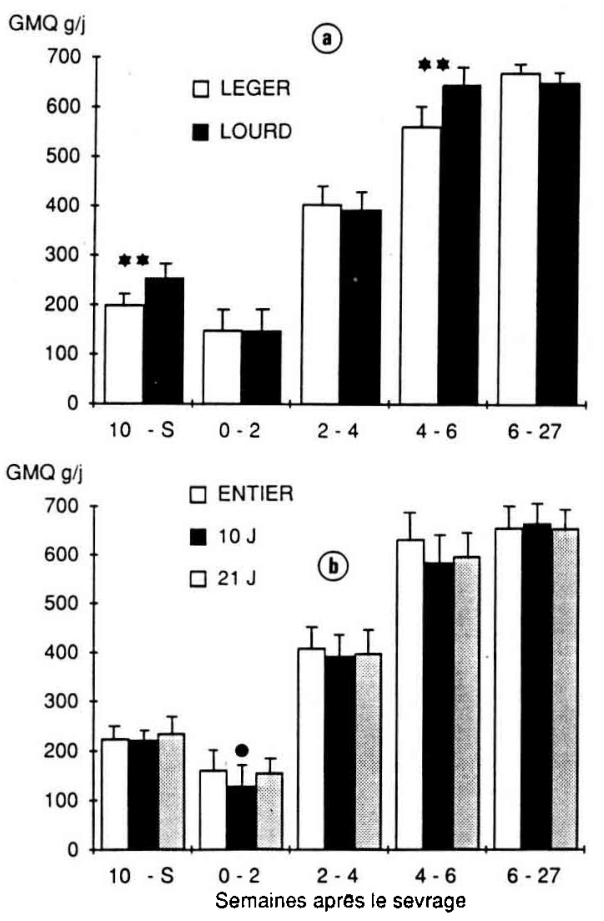

Fig 1. Effet du poids à $10 \mathrm{j}$ (a) et de l'âge à la castration (b) sur la croissance moyenne journalière $(G M Q)$ des animaux à différentes périodes. 10-S : entre $10 \mathrm{j}$ et le sevrage; $6-27$ : entre 6 semaines et $27 \mathrm{~kg} ;{ }^{* *}$ : Effet poids à $10 \mathrm{j}, P<0,01$; - : Effet castration à $10 \mathrm{j}, P<0,10$. 
Tableau II. Poids moyen des animaux aux différents stades de l'expérience, et performances de la naissance à $27 \mathrm{~kg}$.

\begin{tabular}{|c|c|c|c|c|c|c|c|c|c|}
\hline \multicolumn{2}{|c|}{ Classe de poids à $10 \mathrm{j}(P)$} & \multicolumn{2}{|l|}{ Légers } & \multicolumn{3}{|c|}{ Lourds } & \multicolumn{3}{|c|}{ Significat Statist } \\
\hline & $E^{2}$ & 10 & 21 & $E^{2}$ & 10 & 21 & Effe & $s^{1}$ & $s \bar{x}$ \\
\hline \multicolumn{10}{|l|}{ Poids, $\mathrm{kg}$} \\
\hline Naissance & $1,238^{a}$ & $1,305^{a b}$ & $1,272^{a b}$ & $1,519^{c}$ & $1,425^{b c}$ & $1,420^{b c}$ & $P^{* *}$ & - & 0,022 \\
\hline $10 j$ & $2,69^{a}$ & $2,66^{a}$ & $2,71^{a}$ & $3,63^{b}$ & $3,58^{b}$ & $3,63^{b}$ & $P^{* *}$ & - & 0,053 \\
\hline Sevrage $(23 \pm 1 \mathrm{j})$ & $5,32^{a}$ & $5,10^{a}$ & $5,37^{a}$ & $6,77^{b}$ & $6,91^{b}$ & $7,07^{b}$ & $P^{\prime *}$ & - & 0,116 \\
\hline Fin d'observation & 27,6 & 26,8 & 27,2 & 26,3 & 27,3 & 26,7 & - & - & 0,229 \\
\hline \multicolumn{10}{|c|}{$\begin{array}{l}\text { Performances }(10 j-27 \mathrm{~kg}) \text { : } \\
\text { Gain de poids }(\mathrm{g} / \mathrm{j})\end{array}$} \\
\hline $\begin{array}{l}10 \text { j-Sevrage } \\
\text { Sevrage à } 27 \mathrm{~kg}\end{array}$ & $\begin{array}{l}203^{a} \\
445\end{array}$ & $\begin{array}{l}213^{a} \\
433\end{array}$ & $\begin{array}{l}212^{a b} \\
428\end{array}$ & $\begin{array}{l}248^{b} \\
433\end{array}$ & $\begin{array}{l}260^{b} \\
428\end{array}$ & $\begin{array}{l}263^{b} \\
422\end{array}$ & $\begin{array}{l}P^{* *} \\
-\end{array}$ & - & $\begin{array}{l}5,9 \\
5,8\end{array}$ \\
\hline \multicolumn{10}{|l|}{ Aliment ingéré $(g / j)$} \\
\hline Sevrage a $27 \mathrm{~kg}$ & 753 & 756 & 753 & 742 & 754 & 753 & - & - & 3,5 \\
\hline \multicolumn{10}{|c|}{ Indice de consommation } \\
\hline Sevrage à $27 \mathrm{~kg}$ & $1,70^{\mathrm{a}}$ & $1,75^{a}$ & $1,78^{\mathrm{ab}}$ & $1,73^{a}$ & $1,78^{\mathrm{ab}}$ & $1,81^{b}$ & - & $\mathrm{C}^{*}$ & 0,03 \\
\hline
\end{tabular}

$1 P$ : Effet poids à 10 jours; $C$ : Effel castration; ${ }^{*} P<0,05 ;{ }^{* *} P<0,01 . S \bar{x}$ : Écart type résiduel. ${ }^{2}$ Animaux entiers. $a, b, c$ : Les valeurs affectées d'une même lettre sur une même ligne ne diffèrent pas significativement entre elles.

castrés, entre 27 et $60 \mathrm{~kg}$; elle est, au contraire, significativement supérieure à celle des animaux castrés entre 60 et 100 $\mathrm{kg}$. Elle est ainsi significativement différente au cours de l'ensemble de la période. Ainsi, les máies entiers sont-ils significativement pius jeunes que les animaux castrés à la fois à $25 \mathrm{~kg}$ et à $100 \mathrm{~kg}$ (tableau III). Ils consomment toujours moins d'aliment (8-10\%) que les castrés, et en conséquence leur indice de consommation est significativement abaissé au cours des 2 périodes considérées, soit 8 et $20 \%$ respectivement. Au contraire, l'âge à la castration est sans effet sur les paramètres zootechniques considérés entre 27 et 100 $\mathrm{kg}$. Enfin, le poids à $10 \mathrm{j}$ a un effet limité sur les performances, mais la vitesse de croissance entre 60 et $100 \mathrm{~kg}$ est mini- male, et l'âge corrigé à 25 et à $100 \mathrm{~kg}$ est maximal pour les porcelets légers et castrés à $10 \mathrm{j}$.

\section{Performances à l'abattage}

On observe un effet significatif de la castration qui augmente le rendement à l'abattage, mais l'âge à la castration est sans conséquence. Les résultats relatifs à la composition corporelle exprimée par les pourcentages de tissus maigres et de tissus gras dans la carcasse sont présentés au tableau IV. On observe un effet significatif du poids et de la castration sur le pourcentage de muscle, significativement plus faible chez les animaux légers que 
Tableau III Influence du poids et de la castration des porcelets sur les performances moyennes au cours de la période de croissance-finition.

\begin{tabular}{|c|c|c|c|c|c|c|c|c|c|}
\hline \multirow{2}{*}{$\begin{array}{l}\text { Classe de poids } \\
\text { a } 10 \mathrm{j}(P) \\
\text { Castration }(C), j\end{array}$} & \multicolumn{3}{|c|}{ Légers } & \multicolumn{3}{|c|}{ Lourds } & \multicolumn{3}{|c|}{ Significat Statist } \\
\hline & $E^{2}$ & 10 & 21 & $E^{2}$ & 10 & 21 & & $t^{1}$ & $S \bar{x}$ \\
\hline \multicolumn{10}{|c|}{ Performances de 27 à $60 \mathrm{~kg}$} \\
\hline Gain de poids kg/j & 0,768 & 0,759 & 0,743 & 0,733 & 0,750 & 0,756 & - & - & 0,003 \\
\hline $\begin{array}{l}\text { Aliment ingéré kg/j } \\
\text { Indice de }\end{array}$ & $2,09^{a}$ & $2,27^{b}$ & $2,26^{b}$ & $2,03^{a}$ & $2,23^{b}$ & $2,23^{b}$ & - & $\mathrm{C}^{\star *}$ & 0,021 \\
\hline consommation & $2,74^{\mathrm{a}}$ & $2,92^{b c}$ & $3,06^{\mathrm{b}}$ & $2,79 \mathrm{ac}$ & $3,00^{\mathrm{b}}$ & $2,97^{b}$ & - & $C^{* *}$ & 0,003 \\
\hline \multicolumn{10}{|c|}{ Performances de 60 à $100 \mathrm{~kg}$} \\
\hline Gain de poids kg/j & $1,037^{\mathrm{ab}}$ & $0,916^{c}$ & $0,964^{b c}$ & $1,102^{a}$ & $0,991^{b c}$ & $0,981^{b c}$ & $P^{*}$ & $C^{* *}$ & 0,012 \\
\hline $\begin{array}{l}\text { Aliment ingéré } \mathrm{kg} / \mathrm{j} \\
\text { Indice de }\end{array}$ & $2,93^{a}$ & $3,13^{\text {bc }}$ & $3,18^{\mathrm{b}}$ & $2,97^{a c}$ & $3,26^{\mathrm{b}}$ & $3,15^{\mathrm{b}}$ & - & $\mathrm{C}^{\star *}$ & 0,029 \\
\hline consommation & $2,85^{a}$ & $3,43^{b}$ & $3,30^{\mathrm{ba}}$ & $2,70^{a}$ & $3,31^{b}$ & $3,25^{b}$ & - & $\mathrm{C}^{\star \star}$ & 0,043 \\
\hline \multicolumn{10}{|c|}{ Performances de 27 à $100 \mathrm{~kg}$} \\
\hline Gain de poids kg/j & $0,894^{a}$ & $0,823^{b}$ & $0,840^{\mathrm{b}}$ & $0,900^{\mathrm{a}}$ & $0,865^{a b}$ & $0,861^{a b}$ & - & $\mathrm{C}^{* *}$ & 0,009 \\
\hline $\begin{array}{l}\text { Aliment ingéré } \mathrm{kg} / \mathrm{j} \\
\text { Indice de }\end{array}$ & $2,49^{a}$ & $2,66^{\mathrm{bc}}$ & $2,69 \mathrm{~b}$ & $2,46^{a}$ & $2,74^{b}$ & $2,67^{b}$ & - & $\mathrm{C}^{* *}$ & 0,022 \\
\hline consommation & $2,80^{a}$ & $3,24^{b}$ & $3,24^{b}$ & $2,74^{a}$ & $3,18^{b}$ & $3,12^{b}$ & - & $\mathrm{C}^{* *}$ & 0,032 \\
\hline Age à $25 \mathrm{~kg}(\mathrm{j})$ & $69,2^{a b}$ & $73,6^{c}$ & $70,2^{\mathrm{bc}}$ & $65,7^{a}$ & $67,1^{a b}$ & $66,7^{a}$ & $P^{* *}$ & $C^{* *}$ & 0,58 \\
\hline Age à $100 \mathrm{~kg}(\mathrm{j})$ & $158,4^{a c}$ & $166,6^{\text {bd }}$ & $162,5^{\mathrm{cd}}$ & $155,6^{\mathrm{a}}$ & $157,4^{\text {ac }}$ & $156,7^{a}$ & $P^{* *}$ & $C^{*}(A)$ & 0,73 \\
\hline $\begin{array}{l}\text { Poids à l'abattage } \\
\text { Aliment total / }\end{array}$ & $102,3^{a}$ & $101,2^{a}$ & $101,0^{a}$ & $104,2^{b}$ & $101,4^{a}$ & $101,2^{a}$ & - & $\mathrm{C}^{* *}$ & 0,200 \\
\hline porc $/ \mathrm{kg}^{3}$ & $203,1^{a}$ & $237,0^{\mathrm{b}}$ & $234,8^{b}$ & $202,1^{a}$ & $239,1^{b}$ & $238,2^{b}$ & - & $C^{* *}$ & 2,03 \\
\hline
\end{tabular}

${ }^{1} \mathrm{P}$ : Effet poids à $10 \mathrm{j} ; \mathrm{C}:$ Effet castration; (A) ${ }^{\circ}:$ Effet âge à la castration $P<0,10 .{ }^{2}$ Entiers. ${ }^{3}$ Corrigé à $100 \mathrm{~kg}$, de 25-100 kg. ${ }^{\text {a; }}$; $c$ : Voir tableau II, " $P<0,05 ;{ }^{* *} P<0,01$.

chez les animaux lourds à $10 \mathrm{j}(P<0,05)$. La castration réduit en moyenne de 3,3 points le pourcentage de muscle. Le poids à 10 jours est, par contre, sans effet sur le pourcentage de tissu gras. Au contraire, la castration augmente significativement ce pourcentage de tissus gras exprimé selon les 2 méthodes. Enfin, la comparaison effectuée à l'aide de la méthode des contrastes ne fait pas apparaître de différence significative entre les données de composition corporelle des animaux cas- trés à $10 \mathrm{j}$ ou à $21 \mathrm{j}$. Cependant, lorsque les pourcentages sont exprimés par les mesures effectuées au Fat-O-iNeater, les animaux castrés à 21 j ont une teneur en tissu maigre de $1 \%$ supérieure à celle des animaux castrés à 10 j et un pourcentage de tissu gras qui varie dans les mêmes proportions, sans que ces différences atteignent le seuil de signification. L'interaction $P \times C$, significative seulement au seuil de $5 \%$ dans le cas du rendement, ne mérite pas d'être interprétée en détail. 
Tableau IV. Influence du poids à $10 \mathrm{j}$ et de la castration sur le pourcentage et le poids de tissus maigres et de tissus gras dans la carcasse.

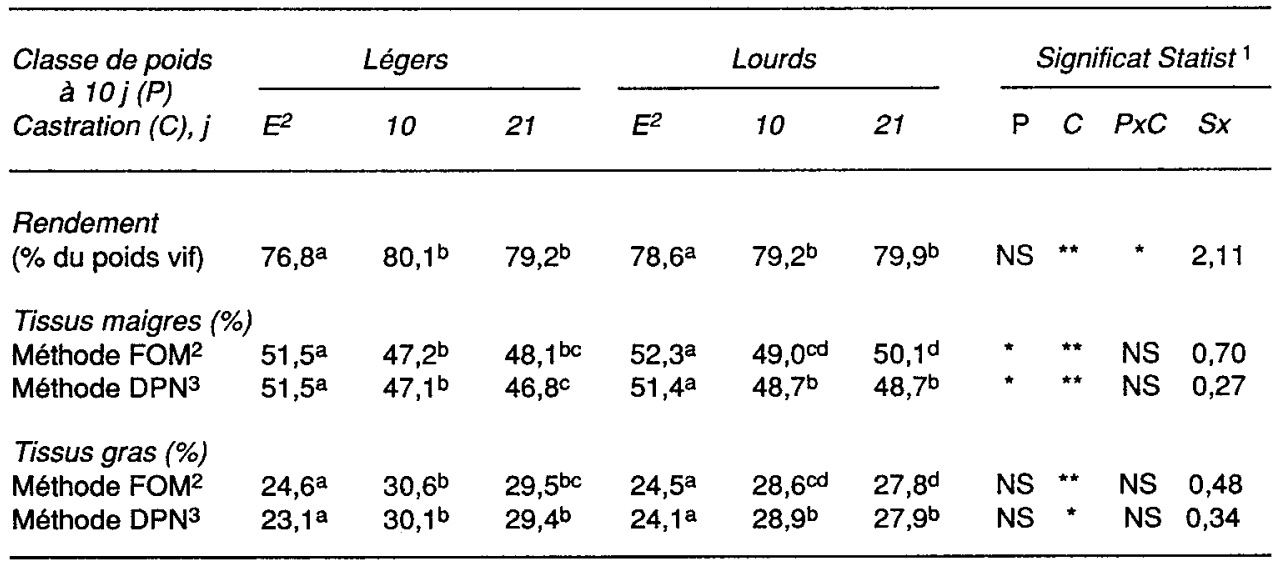

(1) $\mathrm{P}$ : Effet poids à $10 \mathrm{j}$; $\mathrm{C}$ : Effet castration; $\mathrm{P} \times \mathrm{C}=$ Interaction poids $\times$ castration; (2) FOM : Mesures linéaires au Fat-O-Meater. (3) DPN : Découpe partielle de la carcasse (Desmoulin et al, 1988) a, b, c : Voir tableau II; $P<0,05$; ** $P<0,01$.

\section{DISCUSSION}

\section{Performances pondérales}

Le poids à la naissance et corrélativement à $10 \mathrm{j}$ des porcelets mâles, affecte de façon importante leurs performances pondérales jusqu'au sevrage à 23 j d'âge. Son influence décroît par la suite, mais la différence d'âge moyen entre les animaux légers et lourds qui est de 4,5 j à $25 \mathrm{~kg}$ est maintenue à $100 \mathrm{~kg}$. L'âge varie alors de 162,5 à 156,5 j respectivement, en accord avec les données de Powell et Aberle, (1980). L'effet du poids à la naissance, peu marqué pendant la période entre 25 et 60 $\mathrm{kg}$ est encore significatif sur la vitesse de croissance pendant la période allant de 60 à $100 \mathrm{~kg}$. Le phénomène contribue à maintenir l'écart d'âge observé à $25 \mathrm{~kg}$ entre les animaux lourds et les animaux légers jusqu'à $100 \mathrm{~kg}$. Par contre, l'effet de la castration sur la vitesse de croissance, quel que soit l'âge auquel elle est pratiquée, est systématique à partir de $60 \mathrm{~kg}$, et il se répercute sur l'ensemble de la période confirmant avec Balun (1973), Bublick et Gerasimov (1973), et Zivkovic et al (1983), la vitesse de croissance supérieure des mâles entiers. Enfin, les animaux castrés consomment plus d'aliment que les animaux entiers, en accord avec Charette (1961); Pay et Davies (1973); Tesanovic et Vovicic (1975); Desmoulin et Bonneau (1979); Walstra (1980); Knudson et al (1985). Ces résultats peuvent au moins en partie être expliqués par une variation importante de la composition du gain de poids : les porcs entiers, moins gras que les castrés, requièrent moins d'aliment par unité de gain de poids vif. 


\section{Proportions de tissus maigres et de tissus gras}

L'augmentation du rendement à l'abattage des porcs mâles castrés, retrouvée dans notre étude, est une donnée classique (Charette, 1961; Desmoulin et Bonneau, 1979). La réduction du pourcentage de morceaux maigres et l'augmentation du pourcentage de morceaux gras, consécutives à la castration et observées par de nombreux auteurs (Knudson et al, 1985), est confirmée. Les animaux de faible poids à $10 \mathrm{j}$ sont aussi les moins riches en tissus maigres à l'abattage, quel que soit le mode d'expression du pourcentage. Ces données confirment les résultats de Powell et Aberle (1980), observant que les animaux nés à faible poids et ayant une vitesse de croissance postnatale ralentie, sont toujours plus gras à $100 \mathrm{~kg}$. La tendance à une réduction du pourcentage de morceaux maigres chez les animaux castrés à $10 \mathrm{j}$, par rapport à ceux castrés à $21 \mathrm{j}$, confirme les résultats observés par Marks et al (1988). Bien qu'elle n'atteigne pas le seuil de la signification statistique $(P<0,10)$, elle paraît intéressante à souligner, même si elle est observée seulement lors de son expression par la méthode de mesures linéaires à l'aide du FatO-Meater. En effet, on sait depuis longtemps que la modification de la courbe de croissance des porcelets pendant le jeune âge entraîne ultérieurement un excès de dépôt gras (Lucas et al, 1959; Boaz et Elsley, 1962). Un tel phénomène a pu jouer au détriment des porcelets légers castrés à $10 \mathrm{j}$ dont les carcasses contiennent la plus forte proportion de tissus gras. Nos données permettent, de plus, de formuler l'hypothèse selon laquelle la réduction de la vitesse de croissance dans le jeune âge entraîne à long terme une réduction de la croissance de la masse musculaire (Powell et Aberle, 1980; Sève et Bonneau,
1986). Elles peuvent être enfin rapprochées des observations de Lefaucheur et Vigneron (1988) montrant une importance décisive des 3 premières semaines de vie postnatale au cours desquelles la différenciation biochimique et histologique des fibres musculaires s'opère. Le traumaticme de la castration accentué chez les animaux de faible poids peut se répercuter sur cette différentiation et affecter la croissance ultérieure globale de la masse musculaire en limitant le développement de certaines fibres.

\section{CONCLUSIONS}

II semble désormais clairement établi que la castration des porcelets mâles, lorsqu'elle est pratiquée pour éviter les odeurs sexuelles dans les viandes, entraîne une diminution de l'efficacité alimentaire et augmente l'adiposité des carcasses à l'abattage. Ce phénomène est accentué lorsqu'elle est pratiquée trop tôt sur des animaux de faible poids. Sur la base de nos résultats, il semble que la castration, pratiquée à 21 j sur tous les animaux mâles, limite les effets néfastes sur la croissance musculaire et optimise la composition corporelle à l'abattage.

\section{REMERCIEMENTS}

Ce travail a été réalisé grâce à une aide de la Commission des Communautés européennes.

\section{RÉFÉRENCES}

Balun J (1973) Effect of age on weight gain after castration of male pigs. Folia Veter 17, 167175 
Boaz TG, Elsley FWH (1962) The growth and carcass quality of bacon pigs reared to different weights at 56 days old. Anim Prod 4, 13-24

Bublick MV, Gerasimov VI (1977) The effect of age at castration and ovariectomy on the performance of pigs. Svinarstvo 27, 29-33

Charette LA (1961) The effect of sex and age of male at castration on growth and carcass quality of Yorkshire swine. Can J Anim Sci 41, 30-39.

Cochran WG, Cox GM (1957) Experimental Design. Wiley Interscience, NY, $661 \mathrm{p}$

Desmoulin B, Bonneau M (1979) Production des viandes de porcs mâles entiers ou castrés chez les types Piétrain ou Landrace Belge. Jour Rech Porcine Fr 11, 113-120

Desmoulin B, Ecolan P, Peiniau P, Melani C (1984) La classification des carcasses de porcs selon la teneur en viandes maigres; possibilités et limites de l'appareil Fat-OMeater danois. Journ Rech Porcine Fr 16, 37-48

Desmoulin B, Ecolan P, Bonneau M (1988) Estimation de la composition tissulaire des carcasses de porcs : récapitulatif des diverses méthodes utilisables en expérimentation. INAA Prod Anim 1, 59-64

Domanski J (1973) Effect of castrating boars at different ages on fattening and carcass quality of bacon pigs. Thesis, University of Poznan, Poland, $48 \mathrm{p}$

Gonzales C, Vecchionacce $H$, Martinez A (1984) Effect of age at castration of piglet on preweaning and postweaning gains and carcass characters. Inf Annual Inst Prod Anim Univ Cent Venezuela 88-89

Grosse F (1963) Der Einfluss des Kastrationsalters auf die Ansatzleistung beim Schwein. Arch Tierernähr 6, 210-230

Jost M (1971) Die Mast von spätkastrierten männlichen Schweinen. Schweiz Landwirtsch Monatsh 49, 96-100

Knudson BK, Hogberg MG Merkel RA, Allen RE, Magee WT (1985) Development comparisons of boars and barrows: growth rate, carcass and muscle characteristics. J Anim Sci $61,789-796$
Lefaucheur L, Vigneron $P$ (1986) Postnatal changes in some histochemical and enzymatic characteristics of three pig muscles. Meat Sci 16, 199-216

Lucas FAM, Calder AFC, Smith H (1959) The effect of early weaning and of various growth curves before $50 \mathrm{lb}$ live weight upon subsequent performance and carcass quality. $J$ Agric Sci (Camb) 53, 136-143

Marks MA, Mabry JW, Seerley RW, Rampacek GW (1988) Comparison of early castration at day 1, 7 or 14 on growth and carcass traits in swine. Livest Prod Sci 20, 269-273

Pay MG, Davies TE (1973) Growth, food conversion and carcass characteristics in castrated and entire male pigs fed three different dietary protein levels. J Agric Sci (Camb) 81, 6568

Powell SE, Aberle ED (1980) Effects of birth weight on growth and carcass composition of swine. J Anim Sci 50, 860-868

Prescott JHD, Lamming GE (1967) The influence of castration on the growth of male pigs in relation to high levels of dietary protein. Anim Prod 9, 535-545

Salmon-Legagneur E, Février R (1959) Influence de la castration sur la croissance du porcelet. C A Acad Agric France, 45, 142

Sève B, Bonneau M (1986) Effet à long terme de l'avancement de la date de sevrage et du poids initial sur les performances et la composition corporelle du porc. Jour Rech Porcine Fr 18, 143-154

Tesanovic D, Jovicic M (1975) The effect of sex and castration on growth rate, food conversion and carcass characters of Large White pigs. Arch Poljopr Nauke Teh 28, 17-24

Walstra P (1980) Growth and carcass composition from birth to maturity in relation to feeding level and sex in Dutch Landrage pigs. Thesis, Res Int Zeist, The Netherlands

Warwick BL, Van Lone EE (1926) The effect of castration on pig at différent ages. $J A m$ Vet Med Assoc 69, 622-630

Zivkovic S, Pantic V, Sijacki N, Kolaric S, Kovcin S, Kostic J, Sreckovic A (1983) The effect of a single treatment with sex steroïds on growth, feed efficiency and carcass composition of uncastrated pigs. Stocarstvo 37, 131142 